\title{
EVALUATING THE ACCURACY OF TOOTH-SUPPORTED VS MUCOSA- SUPPORTED 3D-PRINTED SURGICAL GUIDE IN DENTAL IMPLANT PLACEMENT (CROSS-SECTIONAL STUDY)
}

\begin{abstract}
Objectives: This study aims to estimate the accuracy of implant insertion using stereolithographic 3D-printed surgical guides; two types were evaluated: tooth-supported and mucosa-supported guides.
\end{abstract}

Materials and methods: 9 patients were enrolled in this study, 5 males and 4 females, mean age: 49.33 years. 12 implants were inserted using tooth-supported guides While 12 implants were inserted using mucosa-supported guides, deviations between the virtual planned implants and the placed implants were calculated after matching the pre- and post-operative CBCT. Matching process was performed using digital software (Blue Sky Plan); angular deviation, deviation at the entry point and apex of the implant were measured. An independentsamples t-test was performed to compare the two groups using SPSS version 25.

Results: The mean angular deviations were $3.67 \pm 1.61$ degrees and $5.46 \pm 2.41$ degrees with the tooth-supported and mucosa-supported surgical guides respectively, and the mean three-dimensional deviations were $0.70 \pm 0.35 \mathrm{~mm}$ and $1.38 \pm 0.41 \mathrm{~mm}$ at the entry point, $0.99 \pm 0.52 \mathrm{~mm}$ and $1.86 \pm 0.51 \mathrm{~mm}$ at the apex, with the toothsupported and mucosa-supported surgical guides respectively.

Conclusions: The results of this study showed that the accuracy of the tooth-supported guide is superior to the mucosa-supported guide.

Keywords: Accuracy, dental implants, stereolithography, surgical guide.
D * Emad Al Toutangy ${ }^{1}$

D Bassel Bard ${ }^{1}$

iD Mohammad Alaa Alzein ${ }^{1}$

Mohammed Yamen Al-Shurbaji Al-Moziek ${ }^{1}$

ORCID IDs of the authors:

E.A.T. $\quad 0000-0003-3726-6366$

B.B. 0000-0002-2195-3076

M.A.A. 0000-0003-1125-9969

A.K. $\quad 0000-0002-3033-4701$
${ }^{1}$ Department of Oral and maxillofacial surgery, Faculty of Dentistry, Damascus University, Damascus, Syria. $\begin{array}{ll}\text { Received } \quad: 27.02 .2020 \\ \text { Accepted } & : 07.05 .2020\end{array}$

How to Cite: Al Toutangy E, Bard B, Alaa Alzein M, Al-Shurbaji Al-Moziek M.Y. Evaluating the Accuracy of Tooth-Supported vs Mucosa-Supported 3d-Printed Surgical Guide in Dental Implant Placement. Cumhuriyet Dent J 2020;23:3:154-159.

*Corresponding Author:

Department of Oral and Maxillofacial Surgery, Faculty of Dentistry, Damascus University, Damascus, Syria.

E-mail: emad931t@gmail.com 


\section{INTRODUCTION}

Oral rehabilitation with implant supported prostheses is one of the most common predictable procedures that has become a more widespread method in daily dental practice. ${ }^{1,2}$ Dental implants have been used since 1960s. ${ }^{3}$ Precise preoperative planning is essential for the clinical success of implant placement, aesthetic outcome and longterm predictability of the future prosthesis. This subject is very important with multiple missing teeth, in which landmarks from adjacent or contralateral teeth are missing. The anatomical variables such as the mandibular canal, mental foramen, submandibular fossa, maxillary sinus and possible lesions in the jaws need to be considered before implant insertion. ${ }^{4}$

Practitioners have generally used panoramic radiographs for implant placement; however, this method of assessment has some disadvantages such as distorted images. ${ }^{5}$ andincapability of displaying the bone width. ${ }^{6}$

Due to the introduction of cone beam computed tomography, preoperative threedimensional planning has become popular. Nowadays new technologies are influencing implant placement procedures and enforce our capability in inserting implants according to the virtual planned location. ${ }^{7}$

Computer-Aided Implantology (CAI), or guided surgery, aims to achieve precise implant positioning, avoid injury of vital anatomical structures, and reduce surgical time especially with flapless approach. ${ }^{8}$, Less morbidity and much more comfort to the patient can be attained..$^{9-12}$ Furthermore, a study has found that guided implant placement could be an alternative to bone grafting procedures. ${ }^{13}$ On the other hand, guided surgery is a sensitive procedure that requires several phases; errors may arise in any phase and lead to the final cumulative error. ${ }^{14}$ These errors result in deviations between planned and inserted implants.

The purpose of the present study is to assess and analyze the clinical reliability of implant placement using two types of 3D-printed guides, including tooth-supported and mucosa-supported guides, and to answer the question about how the type of guide support affects the efficacy and accuracy of implant insertion.

\section{MATERIALS AND METHODS}

An approval of the Scientific Research Committee of Damascus University has been obtained, dated on 3/9/2018 with an ID: 3387 for this crosssectional study.

The study was carried out at the Department of Oral \& Maxillofacial Surgery, Faculty of Dentistry, Damascus University.

Nine patients (5 males and 4 females) were enrolled in this study. 24 implants (implant direct - legacy3) were inserted to replace missing teeth. Patients with poor oral hygiene, systemic conditions, previous irradiation therapy, habit of smoking, bone deficiencies and parafunctional habits were excluded.

All patients have signed written informed consents.

In this study the steps below were followed:

1- A radiographic template was fabricated using a radiopaque acrylic material (Acryline X-ray dvt Anax dent) which meets the aesthetic and prosthetic demands. In the case of totally edentulous patient, the radiographic template was created from the existing denture.

2-Data acquisition: a primary CBCT scan of the patient's jaw was performed using (Vatechpax I3d), and the radiographic templates were included in the scans. All the CBCT data was transformed into Digital Imaging and Communications in Medicine (DICOM) files. An impression of the patient's arches was taken, poured and scanned by a lab scanner (MEDIT T300) to produce a (STL) file.

(DICOM) and (STL) files were imported to computer software (Blue Sky Plan - version 4.2.5 - United States) and were merged together.

3- Digital 3D planning: with Blue Sky Plan, implants were virtually planned while taking the anatomical landmarks and prosthetic desire into consideration. A surgical guide was also virtually designed (Figure 1). 

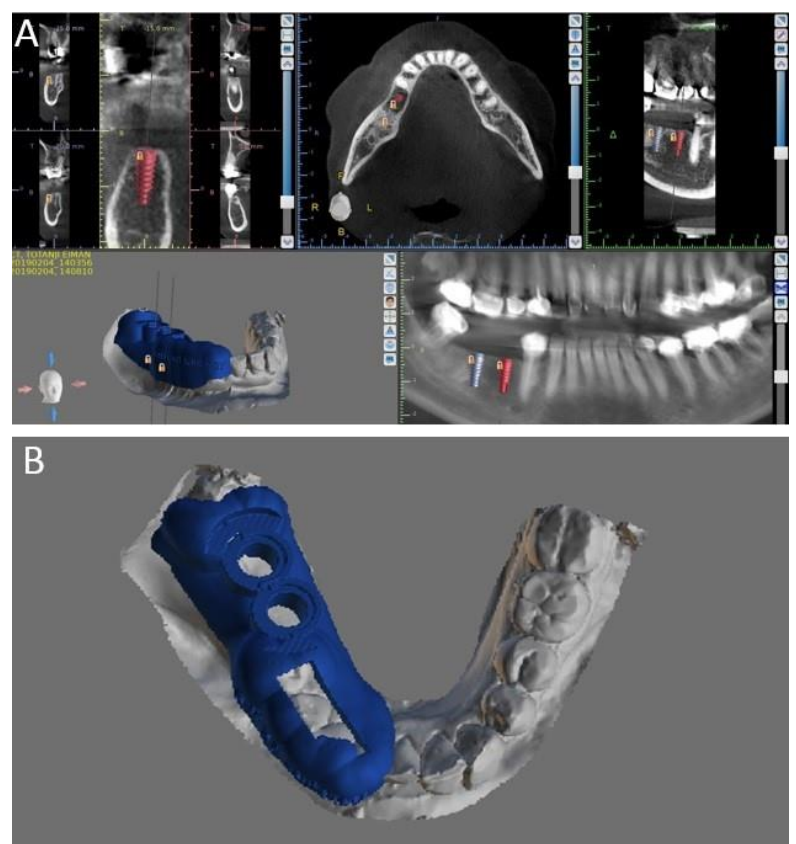

Figure 1: (a) virtual planning of the implants. (b) Virtual design of the surgical guide

4- Surgical guide fabrication: Stereolithography process was used to create the surgical guide from virtual 3D model via 3D printer (ZENITH, Dentis, Daegu, Korea). The angulation and position of each implant as planned with Blue Sky Plan was transferred to the 3D printed surgical guide.

5- Surgical phase:all surgical steps were performed by the same surgeon under local anesthesia using Lidocaine with epinephrine as a vasoconstrictor. (Figure 2A) (Figure2B).

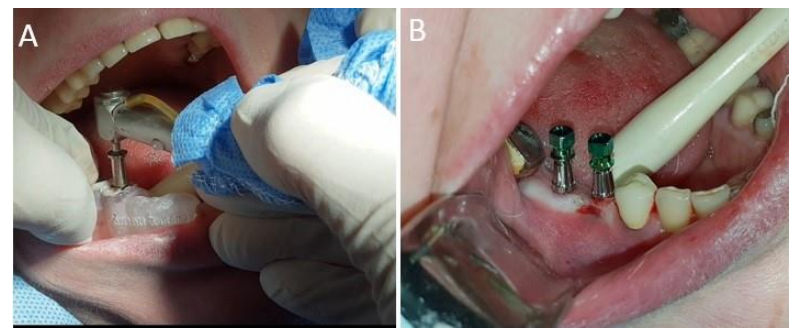

Figure 2: (a) Drilling through the surgical guide. (b) Postoperative view of the inserted implants.

6- Secondary CBCT scan of the patient's jaw was performed using the same primary CBCT parameters.

7- Superimposition of the preoperative and the postoperative CBCT scans was done using Blue Sky Plan (Figure 3), (Figure 4).

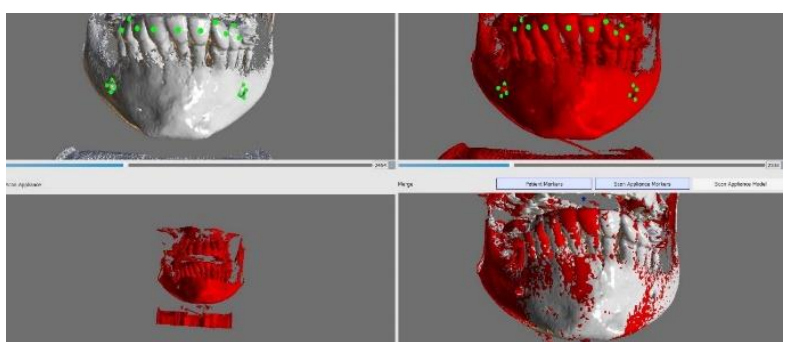

Figure 3: This figure shows the superimposition process of the preoperative and post-operative CBCT.

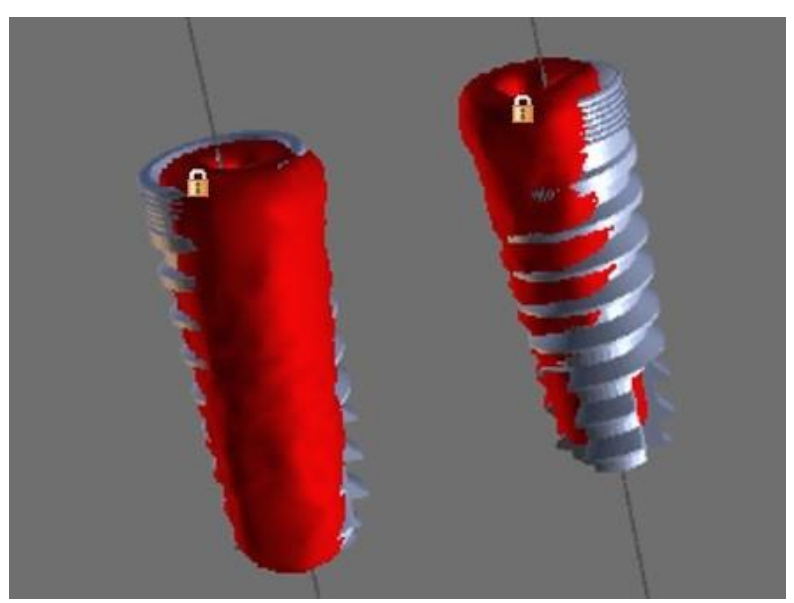

Figure 4: Placed implants represented by red color and planned implants represented by grey color.

8- Calculation of the deviations between virtual and placed implants was done. The deviations were measured at the level of entry point and apex of the implant. Angular deviation was measured as well.

\section{Statistical Analysis}

An independent samples t-test was performed to compare the two groups using SPSS version 25.

\section{RESULTS}

No complications occurred during implant placement.12 implants were placed using toothsupported surgical guides (group 1) and 12 were placed using mucosa-supported surgical guide (group 2).

Data are mean \pm standard deviation. An independent samples t-test was performed to determine if there were statistically significant differences in angular and lateral deviations between the two mentioned groups; results were considered significant at $\mathrm{p}<0.05$.

The mean angular deviations between planned and inserted implants were $3,67^{\circ} \pm 1.61^{\circ}$ and $5.46^{\circ} \pm 2.41^{\circ}$ with the tooth-supported and mucosa-supported surgical guides respectively. The mean lateral deviations were $0.70 \pm 0.35 \mathrm{~mm}$ 
and $1.38 \pm 0.41 \mathrm{~mm}$ at the entry point, $0.99 \pm 0.52$

tooth-supported and mucosa-supported surgical $\mathrm{mm}$ and $1.86 \pm 0.51 \mathrm{~mm}$ at the apex, with the guides respectively. (Table 1)

Table 1. The means, standard deviations, minimum and

\begin{tabular}{lccccccc}
\hline \multirow{2}{*}{ Variables } & $\begin{array}{c}\text { Type of } \\
\text { support }\end{array}$ & Mean & SD & Min & Max & \multicolumn{2}{c}{ 95\% CL } \\
\hline \multirow{2}{*}{ Deviation at entry point $(\mathrm{mm})$} & tooth & 0.70 & 0.35 & 0.30 & 1.50 & 0.48 & 0.92 \\
& mucosa & 1.38 & 0.41 & 0.70 & 2.20 & 1.12 & 1.63 \\
Deviation at apex $(\mathrm{mm})$ & tooth & 0.99 & 0.52 & 0.40 & 2.00 & 0.66 & 1.33 \\
& mucosa & 1.86 & 0.51 & 0.70 & 2.70 & 1.53 & 2.18 \\
Angular deviation $\left(^{\circ}\right)$ & tooth & 3.67 & 1.61 & 1.00 & 6.00 & 2.64 & 4.69 \\
\hline
\end{tabular}

maximum deviations of the angular deviation and of the linear deviations determined at the level of entry point and implant apex.

A Significant difference of $1.79^{\circ}$ (95\% CL, 0.06 to 3.53), $t=2.141, p=0.044$ was found between the two groups for the angular deviation. For the deviation at the entry point there was a significant difference of $0.68 \mathrm{~mm}$ (95\% CL, 0.35 to 1.00$), t=4.347, p<0.001$ between the two groups. There was also a significant difference of $0.87 \mathrm{~mm}(95 \% \mathrm{CL}, 0.43$ to 1.31$), t=4.100, p<0.001$ between the two groups for the deviation at the apex. (Table 2)

Table 2: An independent-samples t-test was performed to make Statistical Comparison of Deviation Differences Between tooth-supported guide group and mucosa-supported guide group, statistical significance level was established at $\mathrm{p}<0.05$

\begin{tabular}{lccccc}
\hline Variables & $\begin{array}{c}\text { Difference in means } \\
\text { between the two } \\
\text { groups }\end{array}$ & $\mathrm{T}$ & P Value & Minimum & Maximum \\
\hline Deviation at entry point & $0.68 \mathrm{~mm}$ & 4.347 & 0.000 & 0.35 & 1.00 \\
Deviation at apex & $0.87 \mathrm{~mm}$ & 4.100 & 0.000 & 0.43 & 1.31 \\
Angular deviation & $1.79^{\circ}$ & 2.141 & 0.044 & 0.06 & 3.53 \\
\hline
\end{tabular}

\section{DISCUSSION}

New technologies in dental implant planning methods have changed our procedures and made it possible to transfer the virtual plan into the surgical field. ${ }^{7} 3 \mathrm{D}$ printed guide is one of the latest technologies in computer-guided surgery. However, deviation from the virtual planning phase is possible. ${ }^{8}$ Several means have been used to evaluate and measure thiserror. ${ }^{15-}$ ${ }^{18}$ Nowadays,Dicom files matching is the standard method used to assess the deviation between the planned and the clinically inserted implants. ${ }^{19}$

To evaluate the accuracy of implant insertion some points should be taken into account:

Deviation at the entry point

Deviation at the apex

Angular deviation. ${ }^{20}$

In an in-vitro study Turbush $\mathrm{et} \mathrm{al.}{ }^{21}$ described the use of Stereolithography to fabricate 30 acrylic resin mandibles: 10 mandibles were modified by adding teeth, and10 mandibles were modified with soft acrylic resin to simulate the mucosa. 150 implants were inserted using bonesupported, tooth supported, mucosa supported surgical guides and superimposition of DICOM files (preoperative and postoperative) were performed using 3D software to calculate the deviations between planned and inserted implants. The mean deviations in position at the neck and apex were $1.00 \pm 0.33 \mathrm{~mm}$ and $1.15 \pm 0.42 \mathrm{~mm}$ for the tooth supported guide, $1.47 \pm 0.43 \mathrm{~mm}$ and $1.65 \pm 0.48 \mathrm{~mm}$, for the mucosa supported guide, $1.08 \pm 0.33 \mathrm{~mm}$ and $1.53 \pm 0.90 \mathrm{~mm}$ for the bone supported guide respectively. However, the angular deviation was $2.26 \pm 1.30$ degrees for the tooth supported guide, $2.29 \pm 1.28$ degrees for the mucosa supported guide, $2.17 \pm 1.02$ degrees for the bone supported guide. This study suggests that there is a slightly better outcome for the tooth supported guide in comparison with the mucosa supported guide. 
Concerning the accuracy of surgical guide for dental implant placement based on the type of supporting tissue, a systematic review. ${ }^{20}$ figured out that tooth-supported guide has better accuracy compared to bone / mucosa-supported guide. This systematic review revealed that the mean deviation at the entry point was $0.84,1.07,1.43$ $\mathrm{mm}$, and at the apex it was $1.15,1.64,1.87 \mathrm{~mm}$ for tooth-, mucosa-, and bone-supported guides, respectively. Comparable results were reported in our study which noted that, regarding the accuracy, tooth supported guides showed lower deviations than mucosa supported guides.

Several factors contribute to the relative inaccuracy of the mucosa supported guide; stabilization and positioning errors have the most significant effect. ${ }^{22}$

The thickness of the mucosa had also been noted as one of the factors contributing to the accuracy of implant insertion with mucosa supported guide, and the deviations increase when the mucosa is thicker. $^{23,24}$

\section{CONCLUSIONS}

The results of this study show that there is a correlation between the guide supporting type and the accuracy of implant placement. The final outcome is the sum of all errors that could happen during the procedure. Furthermore, the mucosasupported guide maybe less accurate than the tooth-supported guide.

\section{ACKNOWLEDGMENT}

This study was carried out at the Department of Oral \& Maxillofacial Surgery, Faculty of Dentistry, Damascus University, which deserves appreciation for the academic support.

\section{CONFLICTS OF INTEREST STATEMENT}

The authors declare no conflicts of interest.

\section{REFERENCES}

1. Ramasamy M, Giri RR, Subramonian K, Narendrakumar R. Implant surgical guides: From the past to the present. J Pharm Bioallied Sci 2013;5:S98.

2. Naziri E, Schramm A, Wilde F. Accuracy of computer-assisted implant placement with insertion templates. GMS Interdiscip Plast Reconstr Surg DGPW 2016;5:2-16.
3. Demirkol N, Demirkol $M$. The Diameter and Length Properties of Single Posterior Dental Implants: A Retrospective Study. Cumhuriyet Dent J 2019;22:276-282.

4. Apaydın BK, Yasar F, Kizildag A, Taşdemir OU. Accuracy of digital panoramic radiographs on the vertical measurements of dental implants. Cumhuriyet Dent J 2018;21:209-215.

5. Yeo DKL, Freer T, Brockhurst P. Distortions in panoramic radiographs. Aust Orthod J 2002;18:92-98.

6. Özalp Ö, Tezerişener HA, Kocabalkan B, et al. Comparing the precision of panoramic radiography and cone-beam computed tomography in avoiding anatomical structures critical to dental implant surgery: A retrospective study. Imaging Sci Dent 2018;48:269275.

7. Skjerven H, Riis UH, Herlofsson BB, Ellingsen JE. In Vivo Accuracy of Implant Placement Using a Full Digital Planning Modality and Stereolithographic Guides. Int J Oral Maxillofac Implants 2019;34:124132.

8. Arısan V, Karabuda ZC, Özdemir T. Accuracy of two stereolithographic guide systems for computer-aided implant placement: a computed tomography-based clinical comparative study. J Periodontol 2010;81:43-51.

9. Campelo LD, Camara JRD. Flapless implant surgery: a 10-year clinical retrospective analysis. Int $\mathbf{J}$ Oral Maxillofac Implants 2002;17:271-276.

10. Brodala N. Flapless surgery and its effect on dental implant outcomes. Int $\mathbf{J}$ Oral Maxillofac Implants 2009;24:118-125.

11. Sclar AG. Guidelines for flapless surgery. J Oral Maxillofac Surg 2007;65:20-32.

12. Becker W, Goldstein M, Becker BE, Sennerby L. Minimally invasive flapless implant surgery: a prospective multicenter study. Clin Implant Dent Relat Res 2005;7:s21-s27.

13. Fortin T, Bosson JL, Isidori M, Blanchet E. Effect of flapless surgery on pain experienced in implant placement using an image-guided system. Int $\mathbf{J}$ Oral Maxillofac Implants 2006;21:298-304. 
14. Cassetta M, Di Mambro A, Giansanti M, Stefanelli

L, Cavallini C. The intrinsic error of a stereolithographic surgical template in implant guided surgery. Int J Oral Maxillofac Surg 2013;42:264-275.

15. Naitoh M, Ariji E, Okumura S, Ohsaki C, Kurita K, Ishigami T. Can implants be correctly angulated based on surgical templates used for osseointegrated dental implants? Clin Oral Implants Res 2000;11:409-414.

16. Al-Harbi SA, Sun AY. Implant placement accuracy when using stereolithographic template as a surgical guide: preliminary results. Implant Dent 2009;18:4656.

17. Van Assche N, Van Steenberghe D, Guerrero ME, Hirsch E, Schutyser F, Quirynen M, Jacobs R. Accuracy of implant placement based on pre-surgical planning of three-dimensional cone-beam images: a pilot study. J Clin Periodontol 2007;34:816-821.

18. Ersoy AE, Turkyilmaz I, Ozan O, McGlumphy EA. Reliability of implant placement with stereolithographic surgical guides generated from computed tomography: clinical data from 94 implants. J Periodontol 2008;79:1339-1345.

19. Cassetta M, Giansanti M, Di Mambro A, Calasso S, Barbato E. Accuracy of two stereolithographic surgical templates: a retrospective study. Clin Implant Dent Relat Res 2013;15:448-459.
20. Tahmaseb A, Wismeijer D, Coucke W, Derksen W. Computer technology applications in surgical implant dentistry: a systematic review. Int J Oral Maxillofac Implants 2014;29:25-42.

21. Turbush SK, Turkyilmaz I. Accuracy of three different types of stereolithographic surgical guide in implant placement: an in vitro study. J Prosthet Dent 2012;108:181-188.

22. D'haese J, Van De Velde T, Elaut L, De Bruyn H. A prospective study on the accuracy of mucosally supported stereolithographic surgical guides in fully edentulous maxillae. Clin Implant Dent Relat Res 2012;14:293-303.

23. Vasak C, Watzak G, Gahleitner A, Strbac G, Schemper M, Zechner W. Computed tomography-based evaluation of template (NobelGuide ${ }^{\mathrm{TM}}$ )-guided implant positions: a prospective radiological study. Clin Oral Implants Res 2011;22:1157-1163.

24. Ochi M, Kanazawa M, Sato D, Kasugai S, Hirano S, Minakuchi S. Factors affecting accuracy of implant placement with mucosa-supported stereolithographic surgical guides in edentulous mandibles. Comput Biol Med 2013;43:1653-1660. 